\title{
Two-dimensional depth-resolved Mueller matrix characterization of biological tissue by optical coherence tomography
}

\author{
Gang Yao and Lihong V. Wang \\ Optical Imaging Laboratory, Biomedical Engineering Program, Texas A\&M University, College Station, Texas 77843-3120
}

Received December 15, 1998

\begin{abstract}
We built a polarization-sensitive optical coherence tomographic system and measured the two-dimensional depth-resolved full $4 \times 4$ Mueller matrix of biological tissue for what is believed to be the first time. The Mueller matrix measurements, which we made by varying the polarization states of the light source and the detector, yielded a complete characterization of the polarization property of the tissue sample. The initial experimental results indicated that this new approach reveals some tissue structures that are not perceptible in standard optical coherence tomography. (c) 1999 Optical Society of America

OCIS codes: $120.2130,170.4500,260.5430,290.7050$.
\end{abstract}

Optical coherence tomography ${ }^{1}$ (OCT) is a noninvasive noncontact imaging technique that can provide high-resolution cross-sectional images of biological tissues. High spatial resolution $(<2 \mu \mathrm{m})$ and high scanning speed (video rate) have been achieved over the past few years.,3 Recently, polarizationsensitive measurements with OCT have received much attention. ${ }^{4-6}$ Results of these studies revealed the importance of polarization as a contrast mechanism. In general, the polarization property of biological tissue is very complicated and cannot be assumed to be linearly birefringent with a fixed fast axis. ${ }^{4,5}$ As is known in polarimetry, Stokes vectors and Mueller matrices ${ }^{7}$ provide complete representations of polarization properties of light and optical samples, respectively. In this Letter we describe a novel polarization-sensitive OCT system for measuring the full $4 \times 4$ Mueller matrix of biological tissue with both depth and lateral resolution.

The Stokes vector $S$ of a light beam is based on six flux measurements with different analyzers in front of the detector: $S=[H+V, H-V, P-M, R-L]^{T}$, where $H, V, P, M, R$, and $L$ are the intensities of the light beam measured with a horizontal linear polarizer, a vertical linear polarizer, a $+45^{\circ}$ linear polarizer, a $135^{\circ}\left(-45^{\circ}\right)$ linear polarizer, a right-circular analyzer, and a left-circular analyzer in front of the detector, respectively. The superscript $T$ transposes the row vector into a column vector. Because of the relationships $H+V=P+M=R+L$, a Stokes vector can be determined by four independent measurements. The Mueller matrix of a sample transforms an incident Stokes vector into the corresponding output Stokes vector. In other words, the Mueller matrix fully characterizes the polarization property of the sample. In the experiment, the Mueller matrix is obtained by measurements with different combinations of polarizers and analyzers. A total of at least 16 intensity measurements are required for a full Mueller matrix.

In an OCT system, the interference signal generated by the light beams from the reference arm and the sample arm is ${ }^{8}$

$$
\begin{aligned}
I_{\mathrm{oct}} & =2 \operatorname{Re}\left[\left\langle\mathbf{E}_{s}\left(l_{s}\right) \cdot \mathbf{E}_{r, A}^{*}\left(l_{r}\right)\right\rangle\right] \\
& =2\left[I_{s, A}\left(l_{s}\right) I_{r, A}(l r)\right]^{1 / 2}|V(\Delta l)| \cos \left(k_{0} \Delta l\right),
\end{aligned}
$$

where $\mathbf{E}_{s}$ denotes the sample electric field; $\mathbf{E}_{r, A}$ denotes the reference electric field with a polarization state of $A ; l_{s}$ and $l_{r}$ are the optical path lengths of the sample arm and the reference arm, respectively; $I_{s, A}$ denotes the light intensity of the sample arm projected onto the polarization state of $A ; I_{r, A}$ denotes the light intensity of the reference arm with the polarization state of $A ; V$ is the temporal coherence function of the field; $\Delta l$ represents the path-length difference between the sample and the reference arms; and $k_{0}$ is the magnitude of the average wave vector. If the sample field reflected from a given depth in the sample were directly measured by a noninterference detector with an analyzer $A$, the flux would be

$$
I_{s, A} \propto I_{\text {oct }}^{2} / I_{r, A} .
$$

A schematic of the OCT system for our studies is shown in Fig. 1. A superluminescent diode with a center wavelength of $0.83 \mu \mathrm{m}$ and a FWHM bandwidth of $20 \mathrm{~nm}$ is used as the light source. The light intensity after the polarizer is $400 \mu \mathrm{W}$. After the light passes through the polarizer, a half-wave plate, and a quarterwave plate, it is split by a nonpolarization beam splitter. The sample beam is focused into the sample by an objective lens (L1) with a N.A. of 0.15 . The reference beam passes through a variable-wave plate and is reflected back. The reflected beams from the reference and the sample arms are coupled into a single-mode fiber and detected by a silicon photodiode. The dynamic range of the system is $110 \mathrm{~dB}$. A single depth scan $(1 \mathrm{~mm})$ takes $4 \mathrm{~s}$ in the current system.

In the experiment we achieve four different incident polarization states, $H, V, P$, and $R$, by rotating the half-wave plate and the quarter-wave plate. 


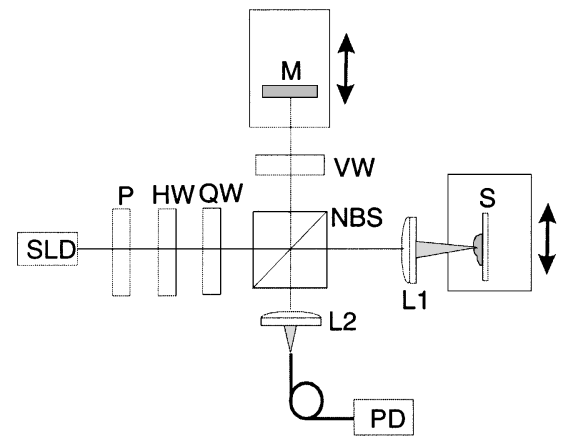

Fig. 1. Schematic of the polarization-sensitive OCT system: SLD, superluminescent diode; P, polarizer; HW, zero-order half-wave plate; QW, zero-order quarter-wave plate; NBS, nonpolarization beam splitter; VW, variablewave plate; M, mirror; L1, L2, lenses; PD, photodiode; S, sample.

For each of these four incident polarization states, we adjusted the variable-wave plate at the reference arm to achieve sequentially the $H, V, P$, and $R$ polarization states. The light intensities of both the source arm and the reference arm are measured for each of the 16 combinations of the polarization states. The source intensity is measured for calibration purposes. The reference intensity is used to convert the OCT signals into a Mueller matrix. We acquire and process a total of 16 polarization-sensitive OCT images to obtain the 16 Mueller matrix images $\left[M_{i j}\right]$ according to relation (2) and the following formula: with their known ideal matrices to within an error of 5$10 \%$. In our setup we find that the error comes mainly from the intensity fluctuation of the interference signal, which is caused by the modulation of the fine spectral structure of the superluminescent diode source.

We performed the initial Mueller matrix measurement on a piece of bone obtained from the head of a yellow croaker fish. The images consist of 100 axial scans with $5-\mu \mathrm{m}$ transverse resolution. The image size is $750 \mu \mathrm{m}$ in the vertical (depth) direction and $500 \mu \mathrm{m}$ in the horizontal direction. The $16 \mathrm{raw}$ images [Fig. 2(a)] show some very interesting patterns. First, there are various degrees of symmetry about the diagonal of the matrix in the morphological appearance. For example, the $H V$ and the $V H$ images are very similar. Second, the depolarization effect can be clearly seen from the differences between the copolarized images $(H H, V V)$ and the cross-polarized images $(H V, V H)$. There are strong cross-polarized signals from some clusters (the red spots in the images) in the $H V$ and the $V H$ images. The corresponding locations in the copolarized images $(H H, V V)$ have strong signals as well. Therefore, these clusters have depolarized the incident light. For most locations other than those depolarizing clusters, the backscattered optical field still preserves most of the original polarization state because the copolarized images are much stronger than the cross-polarized images.

The processed $4 \times 4$ Mueller matrix images are shown in Fig. 2(b). The effect of the beam splitter is

$$
\left[\begin{array}{cc}
H H+H V+V H+V V & H H+H V-V H-V V \\
H H-H V+V H-V V & H H-H V-V H+V V \\
2 H P+2 V P-M_{00} & 2 H P-2 V P-M_{01} \\
2 H R+2 V R-M_{00} & 2 H R-2 V R-M_{01}
\end{array}\right.
$$

$$
\left.\begin{array}{c}
2 R H+2 R V-M_{00} \\
2 R H-2 R V-M_{10} \\
4 R P-2 R H-2 R V-M_{20} \\
4 R R-2 R H-2 R V-M_{30}
\end{array}\right]
$$

where $M_{i j}$, a two-dimensional tomographic image, represents the Mueller matrix element of the $i$ th row and the $j$ th column. The symbols consisting of doublepolarization states represent an OCT measurement, with the source polarization state denoted by the left letter and the reference polarization state denoted by the right letter. For example, $H V$ refers to an OCT image acquired with an $H$-polarized incident field and a $V$-polarized reference field. We can prove matrix (3) by performing matrix operations on the Mueller matrices of the source polarizers, the sample, and the detection analyzers.

The OCT system is carefully calibrated and validated. The four incident polarization states, as well as the four reference polarization states associated with each incident polarization state, are examined in terms of polarization purity. The polarization purity is defined as $I_{\min } / I_{\max }$, where $I_{\max }$ is the signal intensity of the designed polarization state and $I_{\min }$ is the intensity of the orthogonal polarization state. The measured $I_{\min } / I_{\max }$ is less than $0.15 \%$ for all the polarization states. The Mueller matrices of simple optical elements measured with this new technique agree removed from the processed Mueller matrix images, which represent the round-trip polarization characteristics of the tissue sample. The Mueller matrix element $M_{00}$ corresponds to a polarization-independent image as acquired by a nonpolarized OCT system. The other Mueller matrix elements $M_{i j}$ are pixelwise normalized by $M_{00}$. The polarization-independent element $M_{00}$ reveals significantly less information than the other elements, as can clearly be seen in Fig. 2(b). The Mueller matrix images look very different from the raw images and also show some interesting morphological patterns. First, there is some symmetry about the diagonal of the matrix among the images: $M_{01}$ and $M_{10}, M_{02}$ and $M_{20}$, and $M_{12}$ and $M_{21}$ are quite similar. Second, strong layered structures can clearly be seen in some of the images, such as $M_{22}, M_{23}$, and $M_{32}$.

A key organic component of the bone matrix is the collagen fibrils, which should produce linear birefringence. However, the effect of the linear birefringence must have been mixed with that of scattering in this biological sample. According to the Mueller matrix theory, for samples of linear birefringence, their Mueller matrix elements $M_{13}$ and $M_{31}, M_{23}$ and $M_{32}$ 


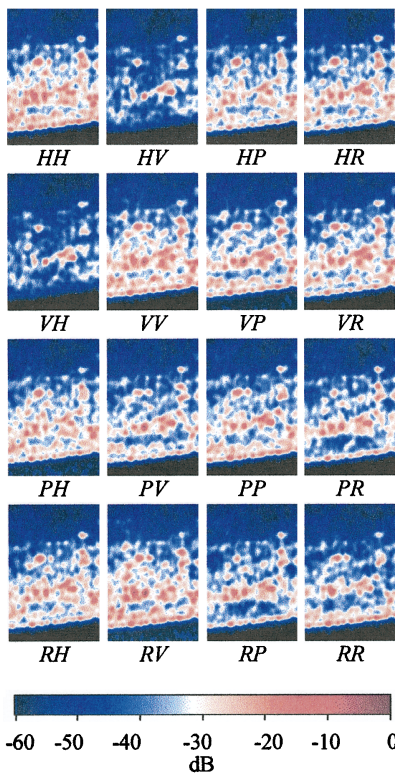

(a) Raw OCT Images.

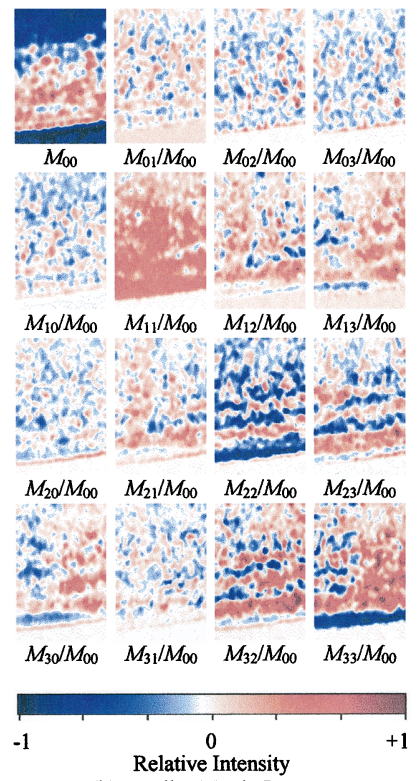

(b) Mueller Matrix Images.

Fig. 2. (a) Raw OCT images with normalized intensity. All the images share the same color map. The size of each image is $750 \mu \mathrm{m} \times 500 \mu \mathrm{m}$. The bottom boundary in each image represents the incident surface of the bone sample. (b) Mueller matrix images. The Mueller matrix images (except $M_{00}$ ) are normalized to $M_{00}$ and share the same color map.

would have identical intensities but different signs. Such antisymmetry is not observed in the images. As shown in a previous study, the depolarization may also result from nonspherical scatterers. ${ }^{6}$ The degree of depolarization has a very complicated dependence on the geometrical and optical parameters of the scatterers. Such a depolarization effect also depends on the polarization state of the incident field because there is much difference between the images acquired with the same reference polarization state and with different incident polarization states. The scatterers with different properties can cluster at different locations within the sample, which may have contributed to the patterns that appeared in the images. In other words, such patterns can provide good indications of spatially localized tissue structures and optical properties. Note that such patterns cannot be captured by nonpolarized OCT imaging and may not be seen by use of incomplete polarization-sensitive OCT imaging.

Definitive interpretations of the images entail precisely stacked three-dimensional histological images with polarization sensitivity. Each slice of the images should be perpendicular to the cross section of the polarization-sensitive OCT images, such that the propagation of light is in the same direction in both measurements. Because of its hardness, bone is difficult to section with a microtome. A frequently used

technique is based on the decalcification of bone preserved by standard fixatives. The decalcified tissue is then embedded, sectioned, and stained. The bone may lose some of its optical properties during this procedure. However, our polarization-sensitive OCT imaging can noninvasively provide depth-resolved polarization information on biological tissue in its native state.

Although our technique can be used to characterize either soft or hard tissue, we measured hard tissue in this experiment because of its stability. To measure soft tissue we will need either to shorten the dataacquisition time or somehow to stabilize the tissue sample at a microscopic scale.

In summary, we have demonstrated a novel imaging technique that combines the advantages of Mueller matrix measurements and optical coherence tomography. This technique can furnish depth-resolved Mueller matrix characterization of native biological tissue either in vivo or in vitro with high spatial resolution. It has a great number of potential applications in biomedicine and material science. Analysis of the Mueller matrix can provide information on the origin of the polarization effect, ${ }^{9}$ which is related to the local anisotropic structure within the sample. As an example, the anisotropic structure of the cardiac muscle is highly correlated with its biological and mechanical properties. Our technique furnishes a tool that is very useful in this area.

Thanks to C. B. Su for experimental assistance. This study was sponsored in part by National Institutes of Health grants R29 CA68562 and R01 CA71980 and by National Science Foundation grant BES-9734491. L. V. Wang's e-mail address is lwang@ tamu.edu.

\section{References}

1. D. Huang, E. A. Swanson, C. P. Lin, J. S. Schuman, W. G. Stinson, W. Chang, M. R. Hee, T. Flotte, K. Gregory, C. A. Puliafito, and J. G. Fujimoto, Science 254, 1178 (1991).

2. X. Clivaz, F. Marquis-Weible, and R. P. Salathé, Proc. SPIE 2083, 338 (1993).

3. A. Rollins, S. Yazdanfar, M. Kulkarni, R. Ungarunyawee, and J. Izatt, Opt. Exp. 3, 219 (1998), www.osa.org.

4. M. J. Everett, K. Schoenenberger, B. W. Colston, Jr., and L. B. Da Silva, Opt. Lett. 23, 228 (1998).

5. J. F. de Boer, T. E. Milner, M. J. C. van Gemert, and J. S. Nelson, Opt. Lett. 22, 934 (1997).

6. J. M. Schmitt and S. H. Xiang, Opt. Lett. 23, 1060 (1998).

7. W. S. Bickel and W. M. Bailey, Am. J. Phys. 53, 468 (1985).

8. Y. Pan, R. Birngruber, J. Rosperich, and R. Engelhardt, Appl. Opt. 34, 6564 (1995).

9. J. J. Gil, and E. Bernabeu, Optik 76, 67 (1987). 